the requirements in ophthalmology consist of : first, case-records ; second, written examination; and third, clinical, laboratory, and oral examinations. The written examination will test the candidate's knowledge of the underlying principles or science of ophthalmology, including anatomy, embryology, physiology, physiological optics, pathology, and relations of the eye to other organs and diseases of the body. Candidates in ophthalmology must submit twenty-five complete case-records, of which ten should be of ocular diseases and defects of varied character, including errors of refraction or muscle balance. The reports should show the reasons for diagnosis and for any operations, the technique of which should be described. The oral examination will include: (1) the external examination of the eye; (2) ophthalmoscopy; (3) estimation of errors of refraction; (4) testing of the ocular movements and of the fields of vision; (5) relations of ocular conditions to diseases of other parts of the body and their treatment; and (6) laboratory examination in histology, pathology, and bacteriology.

.The American College of Surgeons aims high both as regards the scientific standard and the personal character and record of the candidate. A qualification such as it provides should do much to elevate the practice of surgery and of the surgical specialities, of which ophthalmology is one. Moreover, it makes for the standardization of surgery, in itself no small gain.

\title{
A Register of the Blind
}

The Advisory Committee appointed by the President of the Local Government Board to assist him in matters concerning the care and supervision or the blind were confronted at the beginning of their labours by the lack of accurate statistical information as to the number and condition of the blind in England and Wales. The matter was at once referred to a sub-committee, and as a result of their enquiries and report efforts are now being made to compile a complete register of all persons who fall under the definition adopted by the Local Government Board, "too blind to perform work for which eyesight is essential."

This register will contain not merely names and addresses, but also particulars of each blind person, which will enable the authorities to form an opinion as to the best means of rendering assistance, by educating, occupational training, pensioning, etc.

The Local Government Board have now issued forms designed to obtain the desired information, and containing a series of questions to be answered on behalf of the blind. It is hoped that all Boards of Guardians, District Nursing Associations, and societies and institutions ministering to the blind, will assist in making the 
register as complete and accurate as possible. All information supplied will be treated as strictly confidential.

It is only by the aid of an up-to-date register that schemes for the welfare of the blind, now in operation or to be initiated, can be made to include all those entitled to benefit thereby.

\section{Glaucoma produced by Homatropin}

In the American Journal of Ophthalmology for September, 1917, there is a reference to a case of acute inflammatory glaucoma produced by one drop of one per cent. homatropin, recorded by Levitt, of Brooklyn. Why the Editor of the Journal has refrained from stating in what periodical Levitt reports his case, if indeed it has appeared in print at all, it is difficult to understand. But so it is ; no reference is given. In Levitt's case it appears that one drop of one per cent. homatropin employed for a fundus examination caused an attack of acute inflammatory glaucoma; but the age of the patient is not stated. All that is stated is that the eye which developed the glaucoma had tension minus one and was myopic.

\section{ABSTRACTS}

\section{I.-GLAUCOMA}

(I) McCaw, J. A.-The colloidal theory of the pathology of glaucoma. Ophthal. Record, June, I9I 5.

(1) McCaw has made some experiments to test the colloidal theory of glaucoma as advanced by Martin Fischer. His observations on the swelling of fibrin and sheep's eyes when placed in acid solutions, lead him to formulate the following conclusions :

An abnormal production or accumulation of acids, or conditions predisposing thereto, exist in all states in which we encounter the development of oedema. Pathologically considered, glaucoma is a local oedema, and, clinically considered, all the symptoms of this disease are referable to the increase of intra-ocular pressure induced by the large amount of water held by the eye. An intense glaucoma can be induced without any circulation whatever, whilst you may have an increase of pressure in the circulating fluids of the eye without any symptom of glaucoma. "This leads us to the conclusion that the cause of glaucoma may well reside in the tissues of the eye, and that it becomes glaucomatous, not because there is more fluid pressed into it, but because through changes in it, it absorbs more 